\title{
Pipeline for Tracking Neural Progenitor Cells
}

\section{Vestergaard, Jacob Schack; Dahl, Anders Lindbjerg; Holm, Peter; Larsen, Rasmus}

\section{Published in:}

Medical Computer Vision. Recognition Techniques and Applications in Medical Imaging

Link to article, DOI:

10.1007/978-3-642-36620-8_16

Publication date:

2012

Link back to DTU Orbit

Citation (APA):

Vestergaard, J. S., Dahl, A. L., Holm, P., \& Larsen, R. (2012). Pipeline for Tracking Neural Progenitor Cells. In Medical Computer Vision. Recognition Techniques and Applications in Medical Imaging: Second International MICCAI Workshop, MCV 2012, Nice, France, October 5, 2012, Revised Selected Papers (pp. 155-164). Springer. Lecture Notes in Computer Science Vol. 7766 https://doi.org/10.1007/978-3-642-36620-8_16

\section{General rights}

Copyright and moral rights for the publications made accessible in the public portal are retained by the authors and/or other copyright owners and it is a condition of accessing publications that users recognise and abide by the legal requirements associated with these rights.

- Users may download and print one copy of any publication from the public portal for the purpose of private study or research.

- You may not further distribute the material or use it for any profit-making activity or commercial gain

- You may freely distribute the URL identifying the publication in the public portal 


\title{
Pipeline for tracking neural progenitor cells
}

\author{
Jacob S. Vestergaard ${ }^{1}$, Anders L. Dahl ${ }^{1}$, Peter Holm ${ }^{2}$ and Rasmus Larsen ${ }^{1}$ \\ 1 Department of Informatics and Mathematical Modelling, Technical University of \\ Denmark \\ 2 Department of Basic Animal and Veterinary Sciences, Faculty of Life Sciences, \\ Copenhagen University
}

\begin{abstract}
Automated methods for neural stem cell lineage construction become increasingly important due to the large amount of data produced from time lapse imagery of in vitro cell growth experiments. Segmentation algorithms with the ability to adapt to the problem at hand and robust tracking methods play a key role in constructing these lineages. We present here a tracking pipeline based on learning a dictionary of discriminative image patches for segmentation and a graph formulation of the cell matching problem incorporating topology changes and acknowledging the fact that segmentation errors do occur. A matched filter for detection of mitotic candidates is constructed to ensure that cell division is only allowed in the model when relevant. Potentially the combination of these robust methods can simplify the initiation of cell lineage construction and extraction of statistics.
\end{abstract}

\section{Introduction}

Tracking of neural stem cells (NSCs) is fundamental in understanding the causes for cell fate outcomes in in vitro cell growth experiments. Previous studies of stem cells have used manually constructed cell lineages of a limited population to analyze, e.g., the developmental potential [7] or the morphological properties during cell division [5] and clearly show the benefit and importance of cell lineage construction. The development of automated methods for cell lineage construction is a key ingredient in processing large amounts of time lapse imagery and extracting meaningful statistics, previously not possible due to the need for extensive manual interaction.

We present a data driven pipeline for tracking pig neural progenitor cells in phase microscopy time lapse imagery using a supervised segmentation method, accommodating for small imprecisions in the manual annotation, and a completely data driven approach to tracking cells between time points. This pipeline enables for segmentation and tracking thousands of cells from a manual annotation of only 288 cells. The contribution includes novel approaches to mitosis detection, automatic correction of segmentation errors and data driven parameter estimation for the cell matching cost function.

The proposed mitosis detector is based on the observation that a NSC about to undergo mitosis becomes circular and moves out of focus of the imaging 
device, making it easily detectable. A similar behavior is observed by [5] for human neural progenitor cells.

Previously, systems aiming to accomplish the same have been proposed, including LEVER [8] incorporating published methods for segmentation and lineaging $[1,2]$. A limitation by this and other systems is the sensitivity to image data with a slightly different appearance. We explore the possibilities of overcoming this limitation by driving the analysis by simple manual annotation of the image data. This allows the segmentation algorithm to adapt to the problem at hand.

Manual annotation of neural progenitor cells are tedious and difficult even for an expert. A single image cannot be annotated without preceding and following images from the time series. The inherent inaccuracy in these annotations are accommodated for by the choice of segmentation algorithm, namely dictionary learning from image patches. This method exploits the property that the textural appearance of neural progenitor cells can be condensed to a number of typical image patches.

Tracking of the cells during the time lapse image sequence is reduced to match the cells between two time frames. This is accomplished by a modification of the bipartite graph formulation of the matching problem proposed by [6] The modifications introduced are 1) restricting topology changes to ensure cell division occur during cell mitosis and 2) acknowledging that segmentation errors are present and minimizing their disruptions to the cell lineage construction.

The methods applied have been chosen based on the problems arising from analysis of an approximately 83 hours time lapse image sequence with 5 minutes between acquisitions. This sequence consists of 1000 phase contrast microscopy images of neural progenitor cells with very irregular shapes and movement patterns. An example of such an image can be seen in Figure 1a. In the following sections the methodology embedded in the proposed pipeline is outlined and results are reported.

\section{Dictionary learning for robust segmentation}

The cell segmentation is based on a trained dictionary of image and label patches [3]. Each intensity patch in the dictionary has a corresponding label patch. The dictionary is build from manually annotated image exemplars by randomly sampling a set of intensity patches with corresponding label patches. In the training phase the aim is to find a dictionary that well represents the image texture and simultaneously have unique label patches. The label patches have the same spatial resolution as the intensity patches and in each pixel they store the probability of the labels in the training set. A label patch that has high probability for one class and low for other classes in each pixel is considered unique. To optimize the dictionary a weighted k-means procedure is employed where weights are updated in each step based on the uniqueness criterion.

Segmentation of an unknown image is computed using the trained dictionary. For each pixel an image patch is extracted and the label patch corresponding 
to the closest match in the intensity dictionary is assigned. The patches are overlapping, so the obtained probabilities are averaged.

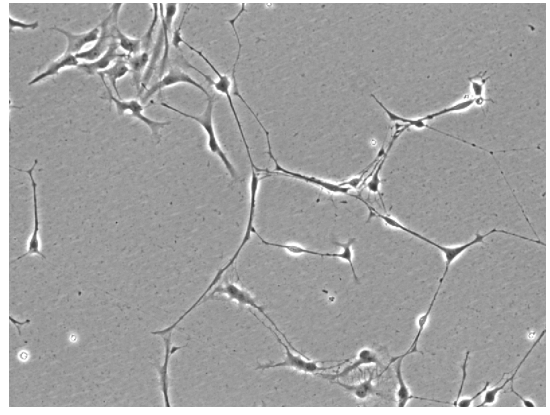

(a)

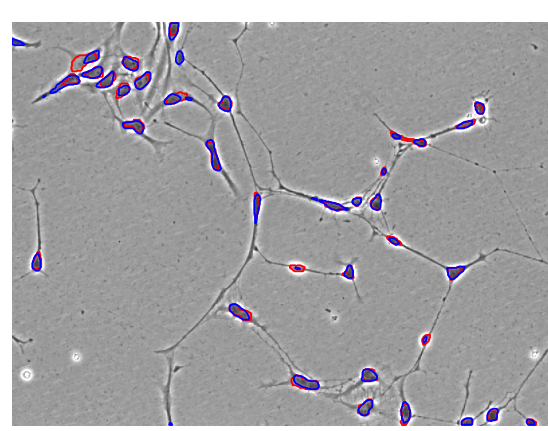

(b)

Fig. 1: a) Phase contrast microscopy image approximately 17 hours into the timeseries. b) Manual annotation (red) together with learned dictionary segmentation (blue) overlaid image.

In this experiment we chose the parameters for the segmentation based on a training and a test set. We had 15 manually annotated image where 8 were used for training and 7 were used for test. Our initial experiments suggested that we needed relatively large image patches, so we chose to downscale the images to half the size giving a spatial resolution of $300 \times 400$. The results of our experiments are shown in Table 1. Dice's coefficient denotes how well the segmentation captures the area and the ratio reported is the number of cells detected versus the number manually annotated. Thus a value above one is over segmentation and below one is under segmentation. The performance improves slightly going from a patch size of 7 to 9 but only little improvement is obtained by going from 9 to 11 . We chose 9 as a good tradeoff between segmentation performance and computation time. It should be noted that it is a difficult task to manually annotate these images, so the results should be seen together with visual inspection of the segmentations as shown in Figure 1.

Table 1: Segmentation results obtained by varying patch sizes.

\begin{tabular}{lcccccc}
\hline & \multicolumn{3}{c}{ Training } & \multicolumn{3}{c}{ Test } \\
Patch size & 7 & 9 & 11 & 7 & 9 & 11 \\
\hline Dice's coefficient & 0.80 & 0.81 & 0.81 & 0.78 & 0.79 & 0.80 \\
$N_{\text {detected }} / N_{\text {true }}$ & 1.13 & 1.05 & 0.96 & 1.25 & 1.12 & 1.02 \\
\hline
\end{tabular}




\section{Mitosis detection}

Visual inspection of the time lapse imagery revealed that when a neural progenitor cell is about to undergo mitosis, it separates itself from the gel in the petri dish, floating up a bit and out of focus. When a cell is out of focus it has a very distinct pattern due to the imaging process.

This pattern can be derived analytically [10], but requires knowledge of the internal microscope parameters, which have not been available in this case. Therefore a model has been constructed from an image containing the pattern of interest. The image and extracted sample is shown in Figures 2a-b.

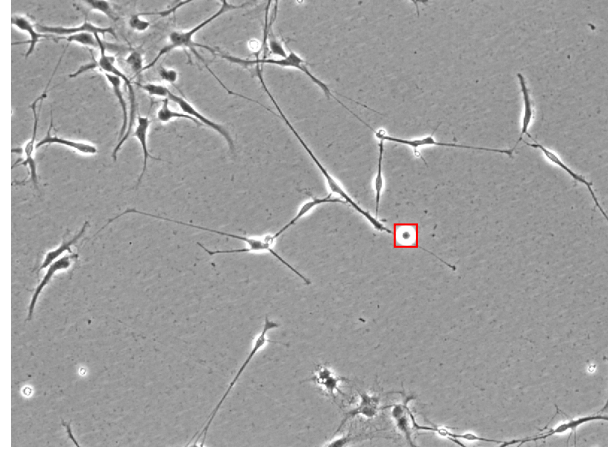

(a) Image

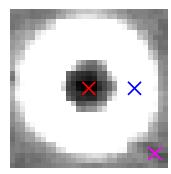

(b) Sample

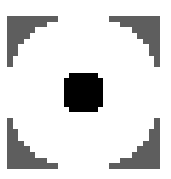

(c) Model

Fig. 2: a) Phase contrast microscopy image with cell in pre-mitotic stage. b) Sample of out-of-focus cell extracted from phase contrast image. Intensity values are extracted from the marked points to transfer the contrast between the halo, center and background to the model. c) Constructed model.

The model is constructed by initializing an image of size $27 \times 27$, which is approximately the same size as the sample. The donut-like center of the sample is modeled by a $27 \times 27$ disk filter, where the hole in the donut is a $7 \times 7$ disk. The intensity values in these three regions are extracted from the sample as marked in Figure 2b. The resulting filter $h$, shown in Figure 2c is normalized by the maximum response from a convolution of the constructed model $\hat{h}$ with the sample $S$, such that $h_{i}=\frac{\hat{h}_{i}}{\max \{\hat{h} * S\}}, i \in\left\{1, \ldots, 27^{2}\right\}$ whereby subsequent filtering can be interpreted as "percentage of perfect response".

The constructed model is used for matched filtering of every phase contrast image. Connected components with a response above 0.9 and an eccentricity below 0.6 is marked as a mitotic candidate. The eccentricity is here the ratio of the distance between the foci of the ellipse and its major axis length. Examples of these detections can be seen in Figure 3.

This detector enables the tracking pipeline to detect and handle cell mitosis, which will be described in Section 4 . 


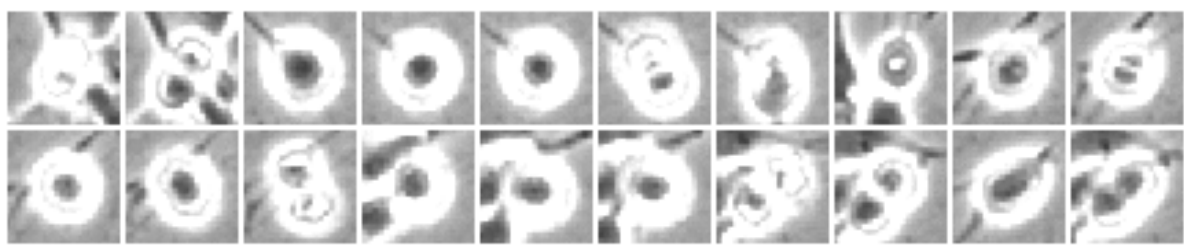

Fig. 3: 20 examples of detected mitotic candidates using the constructed model.

\section{Tracking}

Finding the best match for each cell between two time points is needed to construct cell lineages. Here we employ a tracking method based on initially segmenting the cells, as described above, and subsequently matching the cells between two frames. This is opposed to integrating segmentation and tracking in a single scheme, such as the model evolution approach $[4,9,11]$ where level sets are leveraged as a framework.

The goal is to match $N$ cells $\left\{\mathcal{C}_{i}^{t-1}\right\}_{i=1}^{N}$ detected at the $t-1$ 'th time point to the $M$ cells $\left\{\mathcal{C}_{j}^{t}\right\}_{j=1}^{M}$ at the $t^{\prime}$ th time point. Each of these cells are described with a feature vector $\mathbf{f}$ of length $K$, such that the feature vector for the $j$ 'th cell at time $t$ will be denoted $\mathbf{f}_{j}^{t}$. Specifically we choose to describe each cell with its $x$ - and $y$-coordinates and area, whereby $K=3$.

To match the cells in a way that accounts for all cell features, the matching of cells between two time points can be formulated as a minimum cost problem. We adopt the formulation of the matching problem suggested by [6] where a bipartite graph with coupled edges is set up to accommodate for topology changes.

Tracking by acknowledging segmentation errors Given the difficulty of the segmentation problem the tracking algorithm needs to accommodate for segmentation errors. The possible four types of segmentation errors are:

1. Undetected cell (false negative).

2. Two cells are mistakenly segmented as a single cell.

3. One cell is mistakenly segmented as two cells.

4. Cell detected where none is present (false positive).

It is assumed that any of these segmentation errors are only temporary, i.e., a cell is only undetected or mistakenly segmented for one or a few consecutive time points.

The model by [6] is modified to honor only the biologically possible topology changes, namely that a cell can only split into two if it is undergoing mitosis. A cell is marked as a mitotic candidate if it in the near-past (15 time points $=75$ minutes) has been detected as in the pre-mitotic stage using the detector described in Section 3. The graph illustrating the possible topology changes between two time points is shown in Figure 4. 


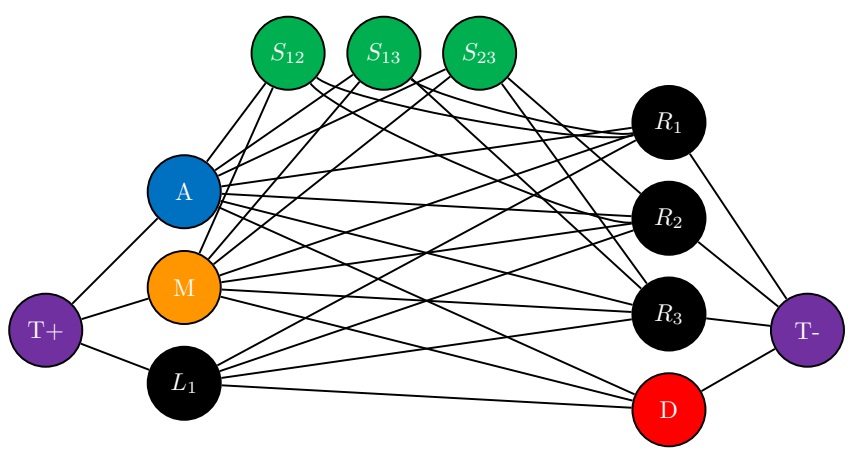

Fig. 4: Graph formulation of the matching problem. In this example there is one cell $M$ detected as undergoing mitosis, wherefore allowed to split. The other cell $L_{1}$ can only move to cells $R_{1}, R_{2}, R_{3}$ or disappear.

Cell merging has not been included in the model as it is not possible for neural progenitor cells to merge with each other. Thereby the possibilities remaining for a cell - not marked as a mitotic candidate - are to move, appear or disappear between frames. The "appear" and "disappear" events include the cases where a cell enters or leaves the image frame, as suggested originally, but also covers the option for a cell to disappear or appear anywhere in the image. This is necessary to accommodate for the segmentation errors listed above.

In the case where a cell from time point $t-1$ is found to disappear, without being near the image border, a phantom (a copy) of the cell is included in the set of cells at time point $t$ and these are coupled as an ordinary "move" event. If no match is found for the phantom for a few time steps (here we choose 2 as the limit), the cell is finally marked as disappeared. For the first two cases listed above, the effect is obviously that the gap between detections is filled with the phantom. For the third and fourth cases, the spurious detection of a new cell in a few images will result in a very short cell track which can easily be detected during post-processing of the lineages. This approach effectively accommodates for the segmentation errors and allows for a robust tracking.

Edge costs Calculation of the edge costs in the graph problem is inspired by [1]. The assigned cost $a\left(\mathcal{C}_{i}^{t-1}, \mathcal{C}_{j}^{t}\right)$ for matching the $i$ 'th cell at time point $t-1$ to the $j$ 'th cell at time point $t$ is the Mahalanobis distance

$$
a\left(\mathcal{C}_{i}^{t-1}, \mathcal{C}_{j}^{t}\right)=\sqrt{\left(\mathbf{d}_{i j}-\boldsymbol{\mu}\right)^{T} \boldsymbol{\Sigma}\left(\mathbf{d}_{i j}-\boldsymbol{\mu}\right)} \quad \text { where } \quad \mathbf{d}_{i j}=\mathbf{f}_{j}^{t}-\mathbf{f}_{i}^{t-1}
$$

from the proposed change feature difference vector $\mathbf{d}_{i j}$ to a reference distribution described by the mean $\boldsymbol{\mu}$ and covariance matrix $\boldsymbol{\Sigma}$.

In [1] this reference distribution is estimated by manually supervising and correcting the tracks from a number of time points. Here we employ a purely data driven approach for estimating this distribution. Specifically, cells are matched over a period of 100 time points with a matching criterion specified as the nearest 


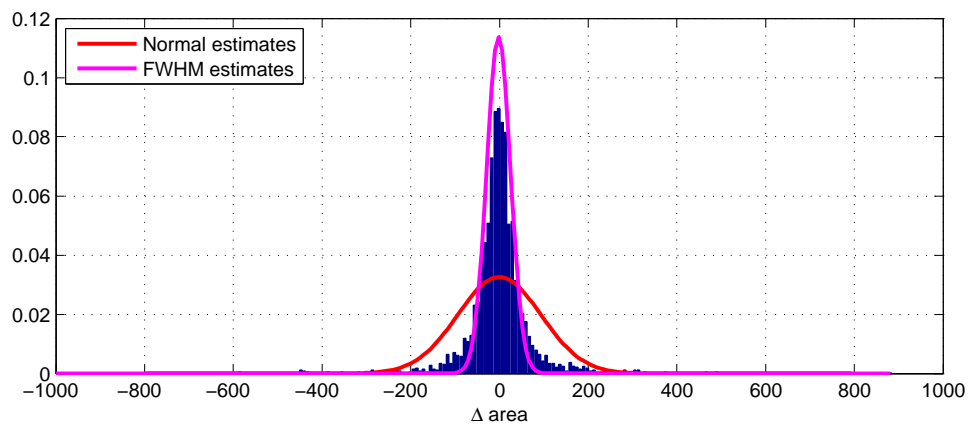

Fig. 5: Illustration of the FWHM principle when estimating parameters for the distribution describing change in area from a one-to-one nearest neighbor tracking.

neighbor, with the constraint that only one-to-one correspondences are accepted, i.e., the nearest neighbor for cell $i$ at time point $t-1$ must also have this cell as its own nearest neighbor. Thereby only the very most obvious matches are included. The feature differences for these $P$ matches are extracted and collected in the $P \times K$ matrix from which the mean $\boldsymbol{\mu}$ and covariance $\boldsymbol{\Sigma}$ of the reference distribution are estimated.

However, with this approach a few erroneous matches are inevitably still included, whereby the parameter estimates are corrupted. To ensure that this does not happen, the principle of full-width-at-half-maximum (FWHM) is used to extract the dominant distribution for each feature difference. Figure 5 illustrates the difference in parameter estimates for the reference Gaussian distribution. It is clear that this is approach is necessary in order to extract viable parameters in a data driven context.

The cost for a cell to appear or disappear is set as the cost of moving from or to a cell with 1) a position of 10 standard deviations of the change in coordinates away and 2) its area set to the mode of the manually annotated cells' area. This forces the model to only let a cell appear or disappear if no suitable match is found in its proximity.

Splitting a cell into two has the cost of moving the cell to the convex hull of the resulting two cells. While there exist $\left(\begin{array}{c}M \\ 2\end{array}\right)$ pairs of potential split candidates, this number can be heavily reduced by selecting only the top $\beta$ percent pairs sorted according to mutual distance as proposed by [6].

\section{$5 \quad$ Results}

The methodology outlined above is applied to the entire time lapse image sequence of 1000 phase contrast microscopy images with 5 minutes between acquisitions. The images were captured using a Nikon BioStation IMQ with a magnification level of 10, an exposure time of $1 / 125 \mathrm{~s}$ and a resolution of $600 \times 800$ 
pixels. The microscope acquires images in a $4 \times 4$ grid, but the images analyzed here are only from a single data point and therefore only $1 / 16$ of the available scene. Thus this analysis should be seen as a proof-of-concept rather than a full analysis.

The mitosis detector described in Section 3 only allows cell division when cells undergo mitosis. Examples of the automatically detected mitotic cells can be seen in Figure 6. From these sequences it is seen that the detector successfully detects the out-of-focus shape characterizing the mitotic candidates, allowing the cell to divide within the near-future (chosen as 15 time points). The examples
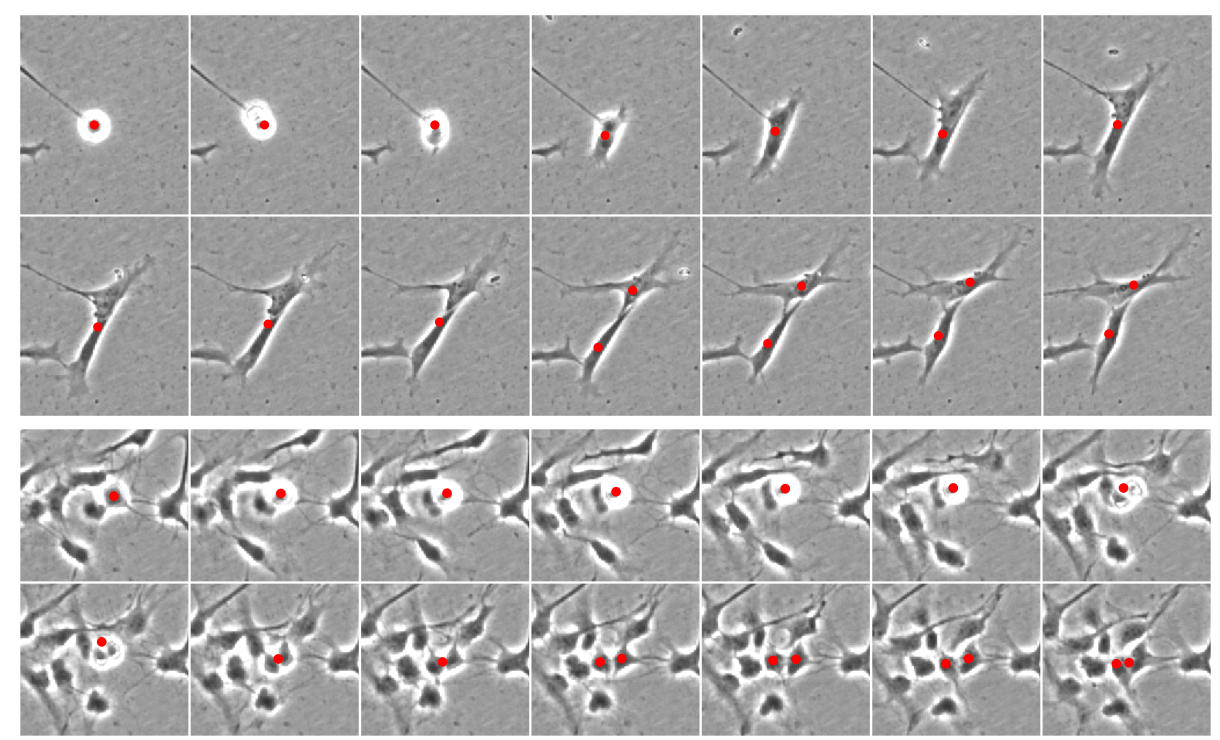

Fig. 6: Two examples of detected mitotic events. Each sequence shows the area of interest from -10 to +3 time points around the detected cell division. The dots indicate the centroids of the detected cells.

illustrate how the mitoses can be detected even in highly confluent areas. A total of 29 mitoses were detected during the entire time lapse sequence using this method. For completeness it should be mentioned that only $62 \%$ were true positive detections.

While the pipeline enables us to follow cells over time, direct interpretation of the cell trajectories is of limited value compared to statistics derived from these. In Figure 7 cell count and step lengths are documented as a function of time. The time series has been divided into ten equally sized periods, wherefore each statistic can be visualized as ten boxes. It is seen that the number of cells increase slightly in the beginning of the period followed by a decrease. Over the entire time period a definite increase in cell count is seen, which is expected given that mitosis occur. 


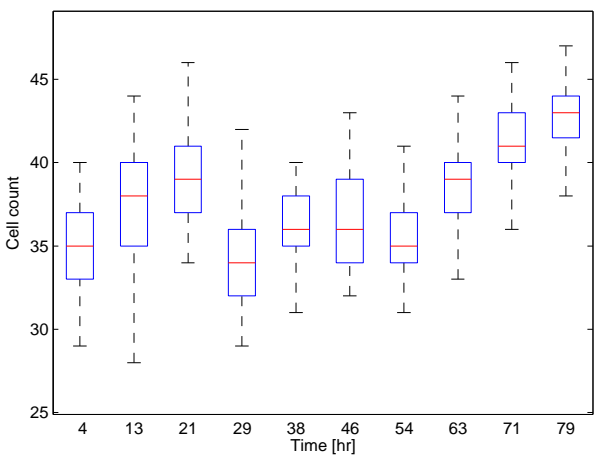

(a) Cell count

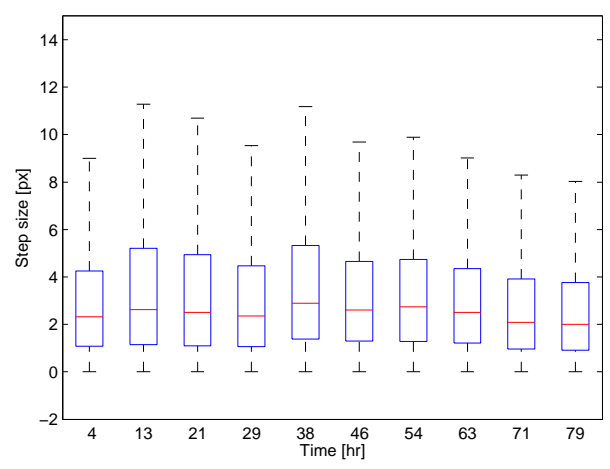

(b) Step lengths

Fig. 7: Simple statistics for the segmented and tracked time series. Each box represents $1 / 10$ of the 83 hour period. The red line indicates the median, the edges of the box the 25th and 75th percentiles and the whiskers extend to the most extreme data points not considered outliers. Outliers are omitted. a) Cell count as a function of time. b) Cell step length between frames as a function of time.

The step length is reasonably constant over the period, except for a reduction in the last 16 hours. Visual inspection of the time lapse imagery confirms that the cells are less mobile towards the end of the time series.

Statistics concerning individual cells' tendency to undergo mitosis, i.e., whether it is the daughters of the same cell that continuously divides, are interesting, but the number of of mitotic events are too few to state anything with regards to this. To answer this question and similar, a large scale study using the proposed pipeline will be carried out in the near future.

\section{Conclusions}

A tracking pipeline based on a few manual annotations has been proposed. The pipeline accommodates for imprecisions in the manual annotation, by choice of segmentation method, and segmentation errors in the tracking model. Parameters for the tracking model were chosen using the principle of full-width-athalf-maximum to ensure meaningful extraction of parameters in a data driven context. A detector for mitotic candidate cells enables the model to restrict topology changes to those valid for a neural progenitor cell.

Validation of the segmentation algorithm was performed using a division into training and test set of 8 and 7 fully annotated images respectively. It was shown that a Dice's coefficient of 0.79 could be achieved while preserving a slight oversegmentation using a dictionary atom size of $9 \times 9$ in an image down sampled to $50 \%$ of the original size. 
The pipeline was applied to a sequence of 1000 phase contrast microscopy images of moving and proliferating neural progenitor cells of very irregular shapes and movement patterns and varying confluence. A total of 30 mitotic events were detected and simple statistics were extracted from the cell lineages. While leaving room for improvements, this work shows that dictionary learning of discriminative image patches combined with a topology change enabling graph formulation is a flexible pipeline that can be applied even to very difficult tracking problems.

\section{$7 \quad$ Acknowledgments}

The authors would like to thank laboratory technician Jytte Nielsen from Department of Basic Animal and Veterinary Sciences, Faculty of Life Sciences, Copenhagen University, for manual annotation of cells and pleasant collaboration.

\section{References}

1. O. Al-Kofahi, R. Radke, S. Goderie, and Q. Shen. Automated Cell Lineage Construction. Cell Cycle, 5(3):327-335, 2006.

2. A. R. Cohen, F. L. a. F. Gomes, B. Roysam, and M. Cayouette. Computational prediction of neural progenitor cell fates. Nature methods, 7(3):213-8, Mar. 2010.

3. A. Dahl and R. Larsen. Learning dictionaries of discriminative image patches. In Proceedings of the British Machine Vision Conference. BMVA, 2011.

4. O. Dzyubachyk, W. a. van Cappellen, J. Essers, W. J. Niessen, and E. Meijering. Advanced level-set-based cell tracking in time-lapse fluorescence microscopy. IEEE Transactions on Medical Imaging, 29(3):852-67, Mar. 2010.

5. T. M. Keenan, A. D. Nelson, J. R. Grinager, J. C. Thelen, and C. N. Svendsen. Real time imaging of human progenitor neurogenesis. PloS one, 5(10):e13187, Jan. 2010.

6. D. Padfield, J. Rittscher, and B. Roysam. Coupled minimum-cost flow cell tracking for high-throughput quantitative analysis. Medical Image Analysis, 15(4):650-668, 2011.

7. R. Ravin, D. Hoeppner, D. Munno, and L. Carmel. Potency and fate specification in CNS stem cell populations in vitro. Cell Stem Cell, 3(6):670-680, 2008.

8. M. Winter, E. Wait, B. Roysam, S. K. Goderie, R. A. N. Ali, E. Kokovay, S. Temple, and A. R. Cohen. Vertebrate neural stem cell segmentation, tracking and lineaging with validation and editing. Nature Protocols, 6(12):1942-1952, Nov. 2011.

9. F. Yang, M. a. Mackey, F. Ianzini, G. Gallardo, and M. Sonka. Cell segmentation, tracking, and mitosis detection using temporal context. Medical Image Computing and Computer-Assisted Intervention-MICCAI 2005, 8(Pt 1):302-9, Jan. 2005.

10. Z. Yin, K. Li, T. Kanade, and M. Chen. Understanding the optics to aid microscopy image segmentation. Medical Image Computing and Computer-Assisted Intervention-MICCAI 2010, pages 209-217, 2010.

11. C. Zimmer, E. Labruyère, V. Meas-Yedid, N. Guillén, and J.-C. Olivo-Marin. Segmentation and tracking of migrating cells in videomicroscopy with parametric active contours: a tool for cell-based drug testing. IEEE Transactions on Medical Imaging, 21(10):1212-21, Oct. 2002. 